\title{
Mean QRS Duration Ventricular Paced
}

National Cancer Institute

\section{Source}

National Cancer Institute. Mean QRS Duration Ventricular Paced. NCI Thesaurus. Code C100390.

The average (mean) duration (time) of the QRS interval, obtained from a set of measurements of the QRS interval while the ventricle rhythm is controlled by an electrical impulse from a mechanical cardiac pacemaker. The QRS interval is defined as the time from the beginning of the QRS complex to the end of the QRS complex, representing the time it takes for the ventricles to depolarize. 